\title{
Diffuse Anterior Retinoblastoma Masquerading as Complicated Cataract - A Case Report
}

\begin{abstract}
Diffuse anterior retinoblastoma $(\mathrm{Rb})$ is a rare variant of this tumor which may involve the anterior chamber of the eye without any detectable mass in the posterior segment. The initial presentation of this tumor as anterior segment pathology may lead to misdiagnosis and erroneous management eventually delaying the definitive therapy. We describe an 8-year-old child presenting with complicated cataract who underwent lens aspiration and later found to have diffuse anterior Rb.
\end{abstract}

Keywords: Anterior retinoblastoma, cataract, diffuse retinoblastoma, retinoblastoma, uveitis

\section{Introduction}

Retinoblastoma $(\mathrm{Rb})$ is the most common intraocular malignancy of childhood, presenting in children $\leq 3-4$ years of age. $^{[1]}$ Classically, $\mathrm{Rb}$ presents as a retrolental mass arising from the retina and visible externally as a white pupillary reflex and grows either towards vitreous (endophytic pattern) or towards choroid (exophytic pattern) or a third pattern demonstrating horizontal growth with minimal vertical growth, giving it a diffuse infiltrating appearance. ${ }^{[2,3]}$ Diffuse anterior $\mathrm{Rb}$ is a rare variant wherein the tumor seeds in the peripheral retina from where cells enter the aqueous humor and implant on the ciliary body with resultant seeding of tumor cells in the iris, lens, trabecular meshwork, and the anterior chamber (AC) without an evident retinal mass. ${ }^{[3]}$

We report a child who presented with complicated cataract, subsequently diagnosed to have diffuse anterior $\mathrm{Rb}$.

\section{Case Report}

An 8-year-old male presented with diminution of vision in the left eye of 8-month duration with pain and redness for 2 months. On examination, the visual acuity was $20 \backslash 20$ in the right eye and light perception in the left eye. The intraocular pressure (IOP) was 10 and 30 $\mathrm{mmHg}$ in the right and left eye (normal IOP: 10-21 mmHg Slit-lamp examination

This is an open access journal, and articles are distributed under the terms of the Creative Commons Attribution-NonCommercial-ShareAlike 4.0 License, which allows others to remix, tweak, and build upon the work non-commercially, as long as appropriate credit is given and the new creations are licensed under the identical terms.

For reprints contact: WKHLRPMedknow_reprints@wolterskluwer.com of the left eye showed cellular reaction, corneal edema and fluffy white exudates stuck to corneal endothelium suggestive of AC inflammation. A white intumescent cataract was remarkable in the left eye precluding the examination of the posterior segment [Figure 1]. The right eye was normal. Ultrasound B-scan of the left eye demonstrated no abnormality in the posterior segment. The general and systemic examination of the child was normal.

With a diagnosis of lens-induced uveitis with secondary glaucoma in the left eye, the child was prescribed oral acetazolamide, topical betamethasone $0.1 \%$, homatropine $2 \%$, and timolol $0.5 \%$ eyedrops. A planned cataractous lens aspiration with intraocular lens implantation was performed, to reduce the IOP, AC inflammation, and visualization of the posterior segment. The fluffy material from AC sent for cytological examination revealed a discretely scattered population of malignant small blue round cells arranged in small clusters, suggestive of retinoblastoma.

A contrast-enhanced computed tomography scan of the orbits showed no intraocular mass lesion. An ultrasound-biomicroscopic examination of the left eye revealed a ciliary body mass involving $360^{\circ}$ of the pars plana region [Figure 2]. Two cycles of neoadjuvant intravenous chemotherapy were administered (carboplatin - $560 \mathrm{mg} / \mathrm{m}^{2}$, vincristine $1.5 \mathrm{mg} / \mathrm{m}^{2}$, and etoposide - $300 \mathrm{mg} / \mathrm{m}^{2}$, given on a single day, 3 weekly). The left eye underwent enucleation with polymethyl methacrylate implant of $18 \mathrm{~mm}$ diameter.

\footnotetext{
How to cite this article: Chhabra $P$, Handa $S$, Singh M, Balyan M, Kumar N, Rohilla M, et al. Diffuse anterior retinoblastoma masquerading as complicated cataract - A case report . Indian J Med Paediatr Oncol 2020;41:923-5.
}

\section{Prashant Chhabra ${ }^{1}$, Sabia Handa ${ }^{2}$, Manpreet Singh', Monika Balyan², Nitin Kumar', Manish Rohilla ${ }^{3}$, Kirti Gupta 4 , Amita Trehan ${ }^{1}$}

${ }^{1}$ Advanced Pediatric Centre, Pediatric Hematology-Oncology Unit, Postgraduate Institute of Medical Education and Research, Chandigarh, India, ${ }^{2}$ Department of Ophthalmology, Advanced Eye Center, Postgraduate Institute of Medical Education and Research, Chandigarh, India, ${ }^{3}$ Department of Cytopathology, Postgraduate Institute of Medical Education and Research, Chandigarh, India, ${ }^{4}$ Department of Histopathology, Postgraduate Institute of Medical Education and Research, Chandigarh, India

Submitted: 10 -Aug-2020 Revised: $27-A u g-2020$ Accepted: 02-Oct-2020 Published: 31-Dec-2020

Address for correspondence: Dr. Amita Trehan, Advanced Pediatric Centre, Pediatric Hematology-Oncology Unit, Postgraduate Institute of Medical Education and Research, Chandigarh, India. E-mail: trehanamita@hotmail. com

Access this article online Website: www.ijmpo.org DOI: 10.4103/ijmpo.ijmpo_385_20 Quick Response Code:

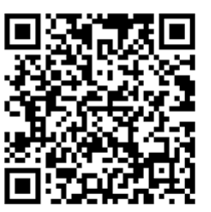




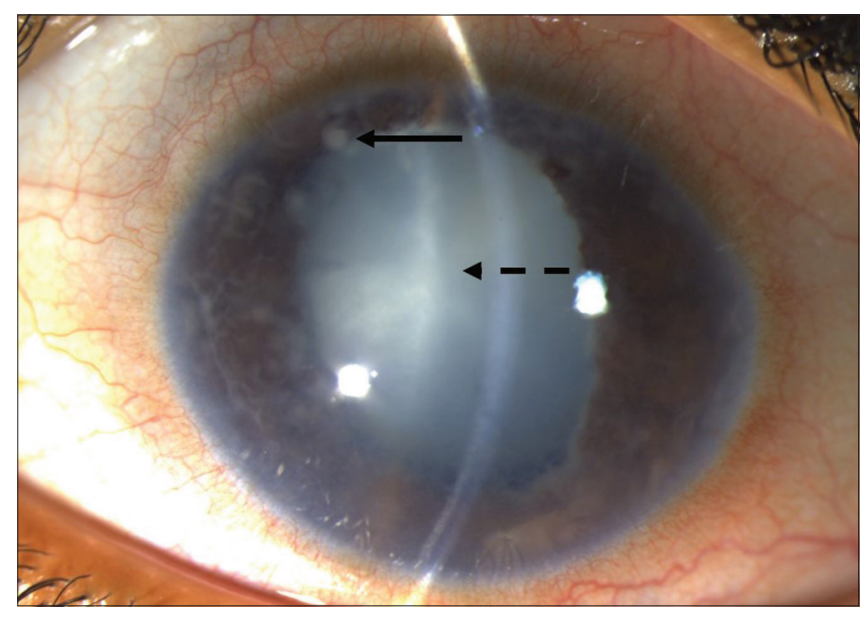

Figure 1: Anterior segment photograph of the left eye of the child showing ciliary and conjunctival congestion. The presence of fluffy white exudates is noted behind the corneal endothelium (solid arrow) along with an intumescent white cataract (dotted arrow)

The histopathology of the eyeball showed normal intraocular structures and optic nerve on gross examination. Microscopic examination revealed floating clusters of tumor cells in AC and a tiny focus of tumor tissue attached to the ciliary body. The tumor had a classical cytomorphology of $\mathrm{Rb}$ showing round blue cells with high nuclear-cytoplasmic ratio, inconspicuous nucleoli and scant cytoplasm. The apoptotic bodies, foci of necrosis, and calcification were also identified. However, no rosettes were seen. The lamina cribrosa and rest of the optic nerve showed no tumor invasion [Figure 3].

Postoperatively, the child received four additional cycles of adjuvant chemotherapy. At present, the child is well, 5 months post completion of treatment.

\section{Discussion}

Approximately $3 \%$ of all $\mathrm{Rb}$ cases present with atypical presentations such as endophthalmitis, secondary glaucoma, pseudouveitis, aseptic orbital cellulitis, cataract, pseudohypopyon, and iris neovascularization. ${ }^{[4,5]}$ These clinical situations pose a diagnostic dilemma and management challenge. ${ }^{[4,5]}$ Diffuse anterior $\mathrm{Rb}$ is a rare variety of $\mathrm{Rb}$ growth pattern, limited to a few case reports. The unilateral involvement in relatively older children of 6-8 years of age, sporadic nature, and frequent misdiagnosis are the hallmarks of diffuse anterior $\mathrm{Rb} \cdot{ }^{[3]}$ In one case report, a germline mutation in $R B 1$ gene was described. ${ }^{[6]}$

Literature review revealed six cases who were initially misdiagnosed owing to atypical presentation and the typical absence of a posterior segment mass lesion. ${ }^{[3,7-11]}$ Garner et al. reported the first-ever case of a 7-year-old child diagnosed as severe granulomatous uveitis. Retinal microscopy revealed two small tumor foci, clinching the diagnosis. ${ }^{[7]}$ Grossniklaus described a child with suspicion of toxocariasis who had evidence of hypopyon and was

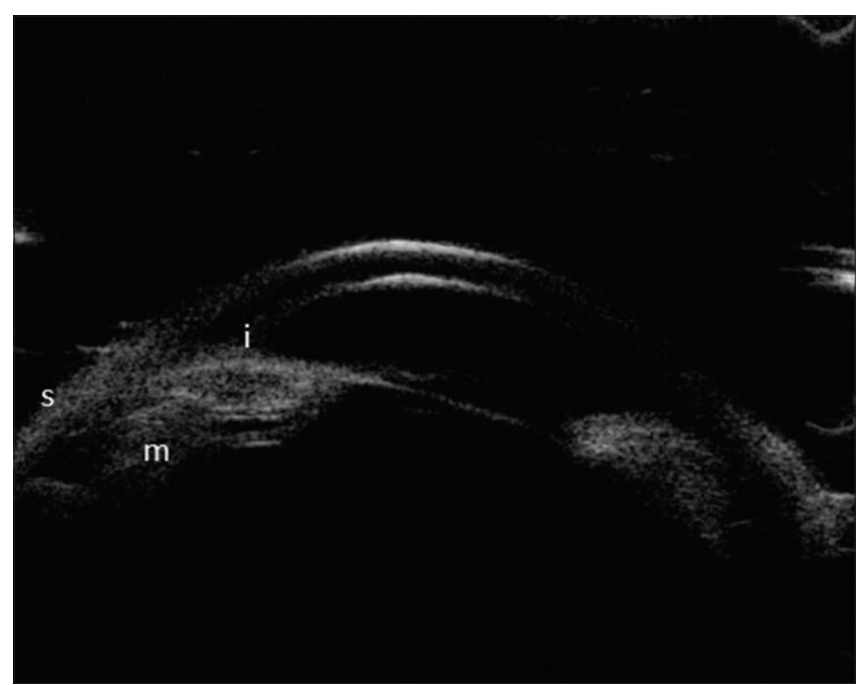

Figure 2: Ultrasound biomicroscopic examination of the left eye showing tumor mass $(\mathrm{m})$ arising from ciliary body adjacent to the sclera (s), abutting the posterior surface of the iris (i)

subsequently diagnosed as $\mathrm{Rb}$ on $\mathrm{AC}$ cytology. ${ }^{[3]}$ Crosby et al. on fundoscopic examination in a 9-year-old child with pseudohypopyon found a small, inferior, peripheral retinal mass. ${ }^{[6]}$ Anterior uveitis with sarcoidosis-like nodules on the iris, ${ }^{[10]}$ hyphema-like presentation, ${ }^{[11]}$ or AC lesions suggestive of exudates ${ }^{[12]}$ have been also described. Our patient presented with a complicated cataract with fluffy white AC exudates which has hitherto been rarely described for diffuse anterior $\mathrm{Rb}$, to the best of our knowledge.

In the absence of an obvious retinal mass, a small tumor focus could be identified in the ciliary body region with ultrasound biomicroscopy in our patient, consistent with other published reports..$^{[9,11,13]}$ However, a definite tumor focus could not be identified in a few reports, thus questioning the cell of origin in such cases, ${ }^{[8,14,15]}$ with the hypothesis that the tumor may be arising from a heterotopic retinal stem cell in the $\mathrm{AC}$ in such cases. ${ }^{[15]}$

The presence of tumor in the ciliary body and vitreous base region leads to shedding of tumor cells in AC through aqueous humor resulting in pseudohypopyon, which is diagnosed as anterior uveitis. The cataract formation in our case is possibly owing to prolonged altered flow dynamics in the circulation of aqueous humor subsequent to the mass arising from the ciliary body. Invasive needle diagnostics are contraindicated in $\mathrm{Rb}$, however, definitive diagnosis in these unusual cases was possible only after a fine-needle aspiration biopsy of AC infiltrate was performed. In our case, fluffy AC exudates were sent for cytology intraoperatively suspecting lens-induced uveitis.

Enucleation with/without systemic chemotherapy and/ or external beam radiotherapy is the treatment for diffuse anterior $\mathrm{Rb}$. Recently, globe salvage has been successfully attempted in four cases with the use of systemic chemotherapy and plaque radiotherapy. ${ }^{[9,11]}$ Rao et al. reported globe salvage in an 18-month-old case with 


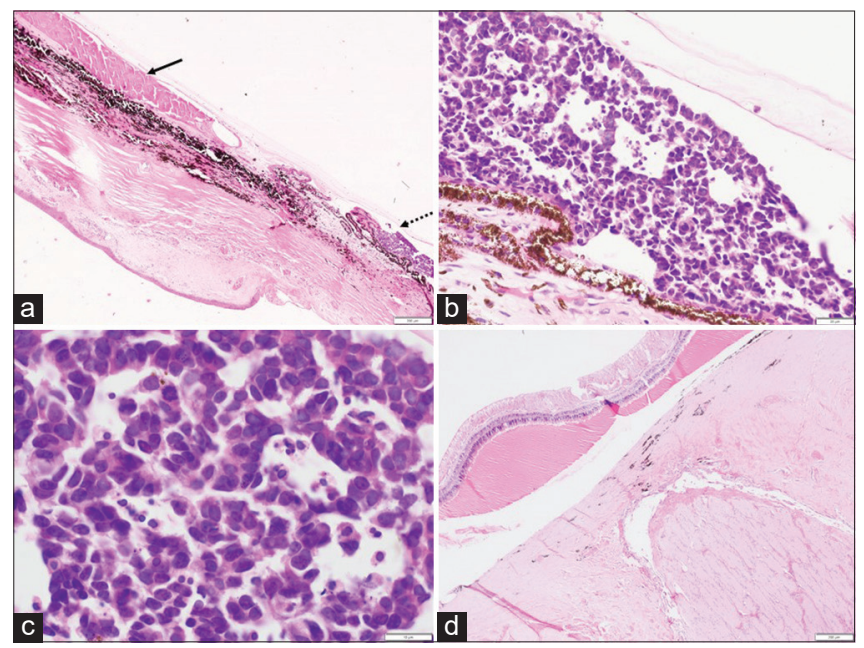

Figure 3: (a) Low magnification showing tumor (dotted arrow) attached to the ciliary body and a degenerated lens (solid arrow). (b) High magnification showing microscopic tumor foci with cells arranged in sheets. (c) Tumor cells showing nuclear molding and apoptotic debris. (d) No infiltration of the optic nerve or lamina cribrosa was noted

administration of systemic chemotherapy, followed by periocular and intravitreal topotecan. ${ }^{[13]}$

In conclusion, diffuse anterior $\mathrm{Rb}$ has unusual ophthalmic presentations without an apparent retinal focus. This rare entity often gets a delayed diagnosis with increased morbidity. The presence of AC exudates with complicated cataract in a child should raise the suspicion for $\mathrm{Rb}$ even in the absence of a posterior segment mass.

\section{Declaration of patient consent}

The authors certify that they have obtained consent from the parents for publication of image (s) and clinical information of the child in the journal. The parents understand that the child's name and initials will not be published and due efforts will be made to conceal the identity.

\section{Financial support and sponsorship}

Nil.

\section{Conflicts of interest}

There are no conflicts of interest.

\section{References}

1. Hurwitz RL, Shields CL, Shields JA, Barrios PC, Gombos DS, Hurwitz MY, et al. Retinoblastoma. In: Pizzo PA, Poplack DG, editors. Principles and Practice of Pediatric Oncology. $7^{\text {th }}$ ed. Philadelphia: Wolters Kluwer Health; 2016. p. 700-25.

2. Jijelava KP, Grossniklaus HE. Diffuse anterior retinoblastoma: A review. Saudi J Ophthalmol 2013;27:135-9.

3. Grossniklaus HE, Dhaliwal RS, Martin DF. Diffuse anterior retinoblastoma. Retina 1998;18:238-41.

4. Balasubramanya R, Pushker N, Bajaj MS, Ghose S, Kashyap S, Rani A. Atypical presentations of retinoblastoma. J Pediatr Ophthalmol Strabismus 2004;41:18-24.

5. Shields CL, Ghassemi F, Tuncer S, Thangappan A, Shields JA. Clinical spectrum of diffuse infiltrating retinoblastoma in 34 consecutive eyes. Ophthalmology 2008;115:2253-8.

6. Crosby MB, Hubbard GB, Gallie BL, Grossniklaus HE. Anterior diffuse retinoblastoma: Mutational analysis and immunofluorescence staining. Arch Pathol Lab Med 2009;133:1215-8.

7. Garner A, Kanski JJ, Kinnear F. Retinoblastoma: Report of a case with minimal retinal involvement but massive anterior segment spread. Br J Ophthalmol 1987;71:858-63.

8. Khetan V, Sudrik S, Singh S, Gopal L, Krishnakumar S. Diffuse anterior retinoblastoma with undetectable retinal involvement. J Pediatr Ophthalmol Strabismus 2011;48 Online: e7-9.

9. Shields CL, Lally SE, Manjandavida FP, Leahey AM, Shields JA. Diffuse anterior retinoblastoma with globe salvage and visual preservation in 3 consecutive cases. Ophthalmology 2016;123:378-84.

10. Kitazawa K, Nagata K, Yamanaka Y, Kuwahara Y, Iehara T, Kinoshita $\mathrm{S}$, et al. Diffuse anterior retinoblastoma with sarcoidosis-like nodule. Case Rep Ophthalmol 2015;6:443-7.

11. Kelly A, Kaufman SC, Ali R, Grajewski A, Anderson J. Rare anterior segment retinoblastoma masquerading as corneal endotheliitis. Eye Contact Lens 2016;42:e1-3.

12. Georgalas I, Koutsandrea C, Paraskevopoulos T, Petrou P. White balls in the anterior chamber: A potentially life-threatening condition. J Pediatr 2016;170:337-e1.

13. Rao R, Honavar SG, Mulay K, Reddy VAP. Eye salvage in diffuse anterior retinoblastoma using systemic chemotherapy with periocular and intravitreal topotecan. J AAPOS 2018;22:235-700.

14. Herwig MC, Hubbard GB, Wells JR, Grossniklaus HE. Diffuse anterior retinoblastoma. Ophthalmologe 2011;108:969-72.

15. Longmuir SQ, Syed NA, Boldt HC. Diffuse anterior retinoblastoma without retinal involvement. Ophthalmology 2010;117:2034-8. 